\title{
An Efficient and Accurate Model for Water with an Improved Non-Bonded Potential
}

Mohamad Mohebifar, Christopher N. Rowley ${ }^{1}$

Department of Chemistry, Memorial University of Newfoundland, NL, Canada

(Dated: 19 May 2020)

A molecular mechanical model for liquid water is developed that uses a physicallymotivated potential to represent Pauli repulsion and dispersion instead of the standard Lennard-Jones potential. The model has three-atomic sites and a virtual site located on the $\angle \mathrm{HOH}$ bisector (i.e., a TIP4P-type model). Pauli-repulsive interactions are represented using a Buckingham-type exponential decay potential. Dispersion interactions are represented by both $C_{6} / r^{6}$ and $C_{8} / r^{8}$ terms. This higher order $C_{8}$ dispersion term has been neglected by most force fields. The ForceBalance code was used to define parameters that optimally reproduce the experimental physical properties of liquid water. The resulting model is in good agreement with the experimental density, dielectric constant, enthalpy of vaporization, isothermal compressibility, thermal expansion coefficient, diffusion coefficient, and radial distribution function. A GPU-accelerated implementation of this improved non-bonded potential can be employed in OpenMM without modification by using the CustomNonBondedForce feature. Efficient and automated parameterization of these non-bonded potentials provides a rational strategy to define a new molecular mechanical force field that treats repulsion and dispersion interactions more rigorously without major modifications to existing simulation codes or a substantially larger computational cost. 


\section{INTRODUCTION}

Molecular mechanical force fields underlie many simulations of materials and biomolecules. These models must effectively capture the significant intermolecular interactions present in a system using computationally-efficient functions. Although these interactions all originate from complex electron-electron, proton-proton, and electron-proton Coulombic interactions, they can be effectively simplified into pairwise electrostatic, London dispersion, and Pauli repulsion interactions.

In most popular molecular mechanical models, electrostatic interactions are described by Coulombic interactions between a set of point charges $(q)$ at atomic centers or positions defined with respect to those atomic centers. Some more elaborate models extend this to include the effects of induced polarization and charge transfer, ${ }^{1-4}$ but force field developers have been largely successful in identifying static charges that capture these complex electrostatic interactions in an effective way.

Dispersion interactions are a ubiquitous, attractive intermolecular force arising through interaction between instantaneous electric moments in neighboring atoms. To a reasonable approximation, this interaction can be approximated as a pairwise sum,

$$
\mathcal{V}_{\text {disp }}(r)=-\frac{C_{6}}{r^{6}}-\frac{C_{8}}{r^{8}}
$$

The magnitude coefficients $C_{6}$ and $C_{8}$ depend on the strength of the dispersion interaction between the interacting atoms. The $C_{6}$ term is the strongest and longest-range term, although quantum chemical analysis and equations of state have found the $C_{8}$ term can also make a substantial contribution to dispersion interactions. The $C_{7}$ and $C_{9}$ terms are less significant for water molecules because of the small magnitude of these coefficients. ${ }^{5}$

Pauli repulsion originates from the overlap of electron density clouds of atoms at close range. As the electron density of atoms follows an exponential dependence, the interaction potential can be described accurately as an exponential decay (i.e., $A \cdot \exp (-b r)$ ), but polynomial terms are often used instead (e.g., $A / r^{12}$ ). In most popular molecular mechanical models, the dispersion and repulsive terms are represented using the Lennard-Jones 12-6 potential $^{6}$,

$$
\mathcal{V}_{n b}(r)=\frac{A_{L J}}{r^{12}}-\frac{C_{6}}{r^{6}}
$$


In this potential, the $A_{L J} / r^{12}$ term is intended to represent Pauli repulsion, while the $-C_{6} / r^{6}$ term represents London dispersion interactions. Higher order dispersion terms are neglected.

These models require the definition of atomic charges and the $A_{L J}$ and $C_{6}$ LennardJones parameters. Generally, the parameters are fit such that the properties predicted by the model are as close as possible to the physical properties of the liquid. Because these intermolecular potentials and simulation algorithms are inexact, the parameters may take on "effective" values that yield accurate predictions of the targeted physical properties but are inconsistent with the true nature of the intermolecular interactions between molecules in the liquids.

The correct strength of dispersion interactions has been a contentious subject in biomolecular simulation. ${ }^{7-10}$ In previous studies by our group, force field dispersion parameters were compared to ab initio values calculated using the eXchange-hole Dipole Moment (XDM) model. This analysis showed that molecular $C_{6}$ coefficients vary widely between force fields, but they were systematically higher than the $a b$ initio values. This trend was attributed to the neglect of higher order dispersion terms from the Lennard-Jones potential.

There are several alternatives to the Lennard-Jones potential that could resolve some of these issues. Buckingham proposed an intermolecular function to describe the interactions of noble gases $^{11}$,

$$
\mathcal{V}_{n b}(r)=A \cdot \exp (-b \cdot r)-\frac{C_{6}}{r^{6}}-\frac{C_{8}}{r^{8}}
$$

Here, the repulsive interaction is described by the $A \cdot \exp (-b \cdot r)$ term, where the $A$ and $b$ parameters define the strength of the repulsion. Dispersion interactions are represented by the $C_{6} / r^{6}$ and $C_{8} / r^{8}$ terms. This potential has several advantages over the LennardJones potential. The exponential term is a more realistic description of Pauli repulsion than the polynomial $A / r^{12}$ term, which is advantageous in simulations where there are frequent repulsive contacts because of strong attractive interactions, high temperatures, or high pressures. Explicit inclusion of the $C_{8} / r^{8}$ term describes the dispersion interaction more realistically as the sum of the $C_{6} / r^{6}$ term and shorter-range $C_{8} / r^{8}$ term, instead of the current practice where all dispersion interactions are effectively included in the $C_{6} / r^{6}$ term.

One issue with the basic form of the Buckingham potential is that the potential becomes infinitely negative as $r \rightarrow 0$. This is a consequence of the exponential term being finite at 
$r=0$ but the dispersion terms becoming infinitely negative. To resolve this issue, Tang and Toennis proposed that the dispersion terms be damped by an incomplete gamma function ${ }^{12}$,

$$
\begin{gathered}
f_{\text {damp }, n}(r)=1-\exp (-\zeta r) \sum_{k=0}^{n} \frac{(\zeta r)^{k}}{k !} \\
\mathcal{V}_{n b}(r)=A \cdot \exp (-b \cdot r)-f_{\text {damp }, 6}(r) \frac{C_{6}}{r^{6}}-f_{\text {damp }, 8}(r) \frac{C_{8}}{r^{8}} .
\end{gathered}
$$

where $\zeta$ is a parameter that corresponds to the strength of the damping.

This non-bonded potential is much more amenable for calculating the long-range component of the $C_{6}$ interaction energy using lattice summation methods. ${ }^{13}$ As the $C_{8} / r^{8}$ term is shorter range than the $C_{6} / r^{6}$ term, a non-bonded cutoff can be applied to this term without neglecting a large component of the dispersion energy.

Although this type of intermolecular potential has found use in models related to chemical physics, molecular simulation of condensed matter still overwhelmingly employ the LennardJones potential to describe these interactions. The foremost barrier to adopting these nonbonded potentials is that it would be necessary to define a complete set of parameters to describe the interaction of each pair of atoms. Determination of optimal parameters has generally been a slow process, where a large set of parameter combinations must be tested. In 2014, Leeping, Martinez, and Pande released the ForceBalance code ${ }^{14}$, which allows optimal parameters for force fields to be determined using a gradient-directed optimization of the parameters.

The ForceBalance code was successfully used to develop new parameters for the TIP3P and TIP4P water models that best described the physical properties of water. A target function was defined based on the enthalpy of vaporization $\left(\Delta H_{\text {vap }}\right)$, density $(\rho)$, isothermal compressibility $\left(\kappa_{T}\right)$, heat capacity $\left(C_{p}\right)$, dielectric constant $\left(\varepsilon_{0}\right)$, and the thermal expansion coefficient $(\alpha)$ of liquid water at standard conditions $(298.15 \mathrm{~K}, 101.325 \mathrm{kPa})$. 


$$
\begin{aligned}
\delta= & b_{1} \cdot\left(\rho_{\text {ref }}-\rho_{\text {calc }}\right)^{2}+ \\
& b_{2} \cdot\left(\Delta H_{\text {vap }, \text { ref }}-\Delta H_{\text {vap }, \text { calc }}\right)^{2}+ \\
& b_{3} \cdot\left(\kappa_{T, \text { ref }}-\kappa T, \text { calc }\right)^{2}+ \\
& b_{4} \cdot\left(C_{p, r e f}-C_{p, \text { calc }}\right)^{2}+ \\
& b_{5} \cdot\left(\varepsilon_{0, r e f}-\varepsilon_{0, \text { calc }}\right)^{2}+ \\
& b_{6} \cdot\left(\alpha_{\text {ref }}-\alpha_{\text {calc }}\right)^{2}
\end{aligned}
$$

The models derived from this optimization, termed TIP3P-FB and TIP4P-FB, provided a significantly improved description of the physical, transport, and structure properties of water. This code provides a viable path to determining appropriate parameters for simulations using the potential defined in Eqn. 5. In this work, we present the development of a new model for liquid water using ForceBalance. This water model is intended to serve as the cornerstone of a new force field based on this improved potential.

\section{COMPUTATIONAL METHODS}

The simulation cell used in the optimization of the model was cubic with an initial side-

length of $18.64 \AA$, containing 215 water molecules. Electrostatic interactions were calculated using the Particle Mesh Ewald method ${ }^{15}$. A correction for the long range component of the $C_{6}$ term of the dispersion interaction was included using the method of Shirts et al. ${ }^{16}$.

All simulations were performed using OpenMM 7.217. Eqn 5 was implemented using OpenMM's CustomNonbondedForce feature that allows the Lennard-Jones interaction potential to be replaced with an alternative equation. For better expression code readability, a Python package, OpenMM Transformer ${ }^{18}$, was used to generate the custom force expressions for Eqn. 5. The ForceBalance code was used to generate optimal parameters using a gradient-directed optimization of the target function defined in Eqn. 7. 


$$
\begin{aligned}
\delta\left(A, b, C_{6}, C_{8}, q_{L P}, l_{L P}\right)= & b_{1} \cdot\left(\rho_{\text {ref }}-\rho_{\text {calc }}\right)^{2}+ \\
& b_{2} \cdot\left(\Delta H_{\text {vap }, \text { ref }}-\Delta H_{\text {vap }, \text { calc }}\right)^{2}+ \\
& b_{3} \cdot\left(\kappa_{T, \text { ref }}-\kappa T, \text { calc }\right)^{2}+ \\
& b_{4} \cdot\left(C_{p, r e f}-C_{p, \text { calc }}\right)^{2}+ \\
& b_{5} \cdot\left(\varepsilon_{0, \text { ref }}-\varepsilon_{0, \text { calc }}\right)^{2}+ \\
& b_{6} \cdot\left(\alpha_{\text {ref }}-\alpha_{\text {calc }}\right)^{2}
\end{aligned}
$$

The enthalpy of vaporization was calculated from the average potential energy of the simulation,

$$
\Delta H_{\text {vap }}=R T-\langle\mathcal{V}\rangle_{\text {liq }} / N_{\text {mol }}
$$

where $N_{m o l}$ is the number of molecules in the simulation and $\mathcal{V}$ is the potential energy.

The thermal expansion coefficient $(\alpha)$ was calculated from,

$$
\alpha=\frac{\left[\langle V \cdot \mathcal{V}\rangle-\langle U \cdot \mathcal{V}\rangle+P\left(\left\langle V^{2}\right\rangle-\langle V\rangle^{2}\right)\right]}{k_{B} T^{2}\langle V\rangle}
$$

where $V$ is the volume of the system.

The isothermal compressibility was calculated from,

$$
\kappa_{T}=\frac{\left\langle V^{2}\right\rangle-\langle V\rangle}{k_{B} T\langle V\rangle}
$$

The dielectric constant was calculated using the relation

$$
\varepsilon_{0}=1+\frac{4 \pi\left(\left\langle M^{2}\right\rangle-\langle M\rangle^{2}\right)}{3\langle V\rangle k_{B} T}
$$

where $M$ is the net dipole moment of the simulation cell.

The diffusion coefficient was calculated from an NVE trajectory of the system using the Einstein relation with the correction for finite-size effects by Yeh and Hummer ${ }^{19}$,

$$
D=\frac{1}{6 t}\left\langle\left|r_{i}(t)-r_{i}(0)\right|^{2}\right\rangle+2.837297 \frac{k_{\mathrm{B}} T}{6 \pi \eta L}
$$


TABLE I. Optimal parameters for a 4-point water model with the Buckingham 6-8 potential.

\begin{tabular}{ll}
\hline parameter & value \\
\hline$A\left(\mathrm{~kJ} \mathrm{~mol}^{-1}\right)$ & $1.60289913 \times 10^{6}$ \\
$b\left(\mathrm{~nm}^{-1}\right)$ & 41.7860722708 \\
$C_{6}\left(\mathrm{~kJ} \mathrm{~mol}^{-1} \mathrm{~nm}^{6}\right)$ & $2.851642 \times 10^{-3}$ \\
$C_{8}\left(\mathrm{~kJ} \mathrm{~mol}^{-1} \mathrm{~nm}^{8}\right)$ & $3.197116358 \times 10^{-5}$ \\
$\gamma\left(\mathrm{nm}^{-1}\right)$ & 35.8967 \\
$q_{O}(e)$ & -1.0517362 \\
$l_{L P}(\mathrm{~nm})$ & 0.106 \\
\hline
\end{tabular}

\section{RESULTS AND DISCUSSION}

This method was used to determine the optimal parameters for the Buckingham-type potential with 6 th and 8th order dispersion (B68) water model. The optimization proceeded for 34 iterations until converging to an optimal set of parameters. These parameters are presented in Table I.

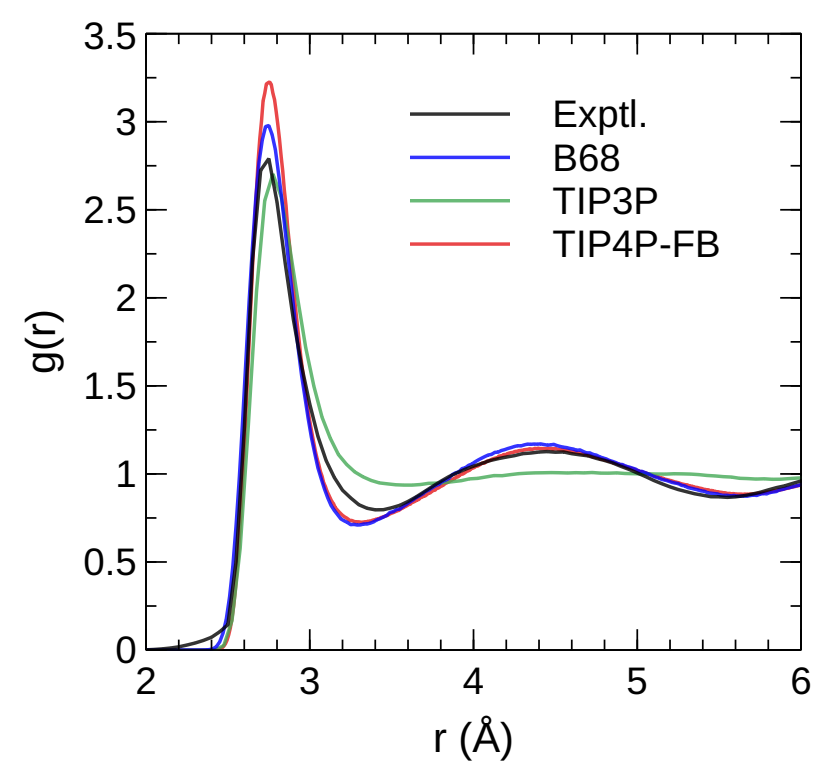

FIG. 1. Radial distribution functions of the TIP3P, TIP4P-FB, and B68 water models compared to the experimental profile from Ref. 20.

This model is in generally good agreement for the targeted physical properties (Table II). The dielectric constants are considerably improved over the TIP3P water model. The 
TABLE II. Properties of liquid water $(298 \mathrm{~K}$ and $101.325 \mathrm{kPa}$ ) predicted by the optimized B68 water model. Density, dielectric constant, enthalpy of vaporization, thermal expansion coefficient, isothermal compressibility, and heat capacity. The properties predicted by the TIP3P and TIP4PFB models, as well as the experimental values, are included for comparison.

\begin{tabular}{lrccc}
\hline Property & TIP3P & TIP4P-FB & B68 & Exptl. \\
\hline$\rho\left(\mathrm{kg} \mathrm{m}{ }^{-3}\right)$ & 983.627 & 995.779 & 997.100 & 997.045 \\
$\varepsilon_{0}$ & 95.809 & 77.288 & 81.040 & 78.409 \\
$\Delta H_{\text {vap }}\left(\mathrm{kJ} \cdot \mathrm{mol}^{-1}\right)$ & 37.108 & 45.210 & 44.293 & 43.989 \\
$\alpha\left(10^{-4} \mathrm{~K}^{-1}\right)$ & 9.049 & 2.442 & 2.609 & 2.572 \\
$\kappa_{T}\left(10^{-6} \mathrm{bar}^{-6}\right)$ & 57.820 & 45.191 & 43.042 & 45.247 \\
$C_{p}\left(10^{-6} \mathrm{cal} \mathrm{mol}^{-1} \cdot \mathrm{K}^{-1}\right)$ & 16.629 & 18.961 & 17.182 & 18.002 \\
\hline
\end{tabular}

O-O radial distribution function of this model is presented in Figure 1, along with those for Lennard-Jones based TIP3P and TIP4P-FB models and an experimental profile determined by X-ray scattering experiments. ${ }^{20}$ The first peak of the $\mathrm{B} 68$ radial distribution function is less steep than TIP4P-FB model, which is in better agreement with the experimental data. This is likely due to the softer exponential repulsive potential in the B68 model. The regions corresponding to the first minimum and second coordination sphere $(r=2.5-5.0 \AA)$ are similar to the experimental data and the TIP4P-FB model.

The temperature dependence of the dielectric constant, thermal expansion coefficient, compressibility, and density of liquid water calculated using these models is presented in Figure 2. The TIP3P potential is a poor model for the temperature dependence of all four properties. The B68 model is in generally good agreement with the experimental values over the full temperature range, with similar performance to the TIP4P-FB water model for most properties, although it overestimates the dielectric constant over the full temperature range and underestimates the compressibility of water at low temperatures.

Historically, part of the justification of the use of Lennard-Jones potentials was that they could be calculated using a small number of multiplication operations, while more complex potentials like Eqn. 5 require the evaluation of additional terms, like the exponential functions. To assess the additional computational cost of the more complex B68 potential in comparison to traditional Lennard-Jones models, benchmark simulations were performed 

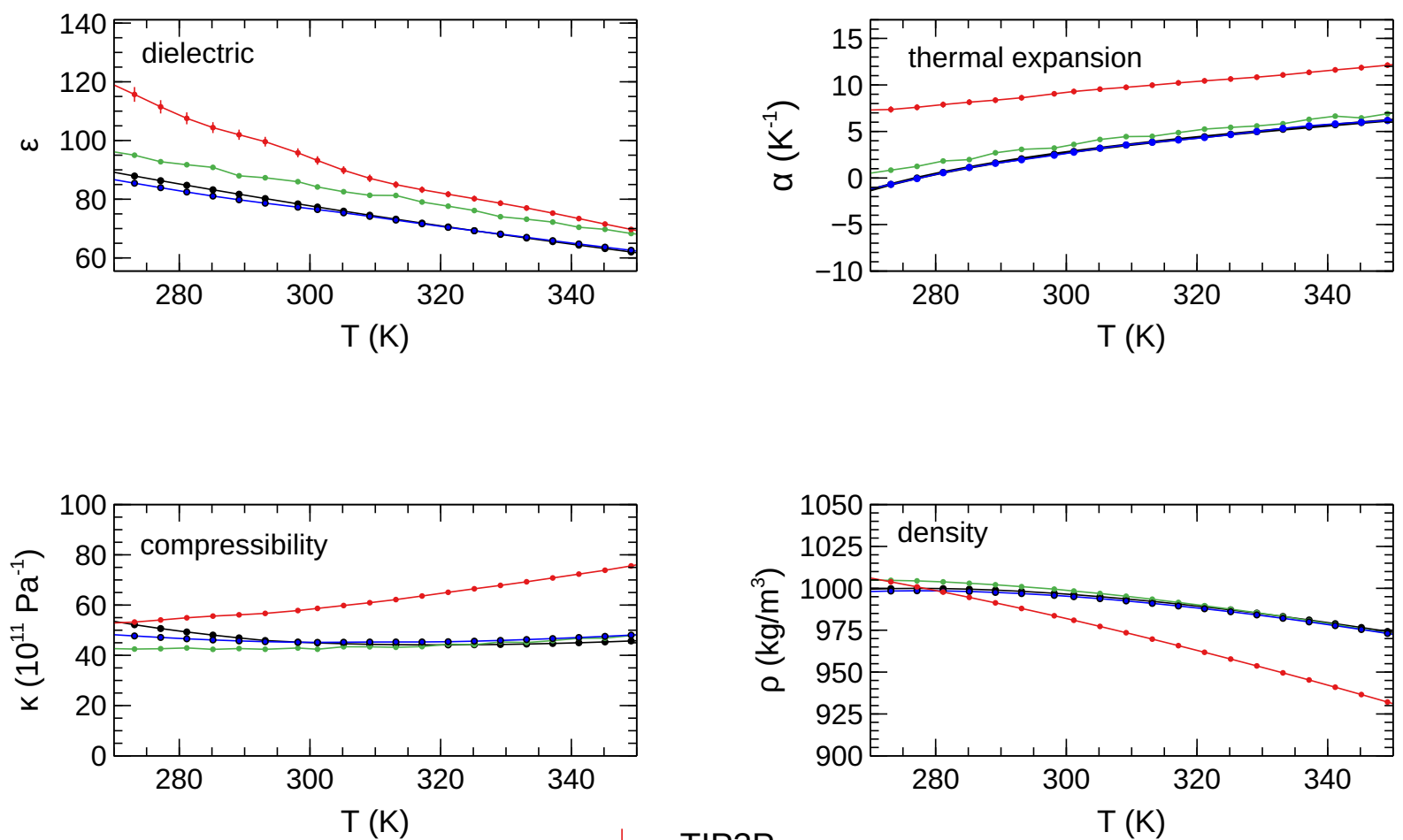

$\begin{array}{ll}+ & \text { TIP3P } \\ + & \text { TIP4P-FB } \\ \div & \text { B68 } \\ - & \text { exptl. }\end{array}$

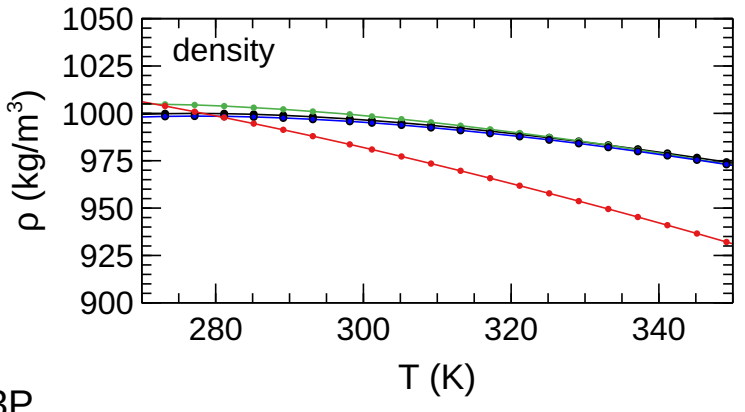

FIG. 2. Temperature dependence of liquid water properties over the temperature range of $270 \mathrm{~K}$ to $350 \mathrm{~K}$ calculated using the TIP3P, TIP4P-FB, and B68 water models compared to the experimental values. Data for TIP3P and TIP4P-FB are taken from Ref. 14.

for a $64 \AA \times 64 \AA \times 64 \AA$ simulation cell containing 8673 water molecules. For comparison, simulations were also performed using TIP4P-FB water model, which employs a conventional Lennard-Jones non-bonded potential. These benchmarks are presented in Table III. When run on the CPU only, the B68 simulations are 10 times slower. This can be attributed to the calculations of exponential terms in the repulsive term and damping functions in the B68 potential, which are computationally-slower operations than the purely polynomial terms in the Lennard-Jones potential. The difference in performance is much smaller GPU implementations; the B68 CUDA simulation runs at $113 \mathrm{~ns} /$ day vs $170 \mathrm{~ns} /$ day for the CPU implementation. The OpenCL implementation shows a similar trend but the performance for both models is slower than the CUDA implementation. We note that this code is generated automatically and more efficient implementations may be possible if these potentials are 
TABLE III. The simulation rate of MD simulations using the B68 model and a Lennard-Jones model (TIP4P-FB) and for a $64 \times 64 \times 64 \AA^{3}$ simulation cell of liquid water. All values are in ns/day using a 2 fs time step. A $3.2 \mathrm{GHz}$ Intel(R) i7-8700 CPU with a Titan Xp NVIDIA GPU was used in these benchmarks.

\begin{tabular}{ccc}
\hline & TIP4P-FB & B68 \\
\hline CPU & 6.17 & 0.61 \\
CUDA & 170.0 & 113.1 \\
OpenCL & 143.0 & 90.7 \\
\hline
\end{tabular}

explicitly added to the simulation codes. Nevertheless, these results show that the LennardJones potential can be replaced with a more physically-motivated potential at an acceptable computational cost.

The $C_{6}$ coefficient of this model very similar to the XDM-calculated value $\left(\mathrm{B} 68 C_{6}\right.$ coefficient: $2.54 \times 10^{-3} \mathrm{~kJ} \mathrm{~mol}^{-1} \mathrm{~nm}^{6} \mathrm{XDM}: 2.57 \times 10^{-3} \mathrm{~kJ} \mathrm{~mol}^{-1} \mathrm{~nm}^{6}$ ) but the $C_{8}$ coefficient is 5 times smaller (XDM: $\left.1.83 \times 10^{-4} \mathrm{~kJ} \mathrm{~mol}^{-1} \mathrm{~nm}^{8} \mathrm{~B} 68: 3.12 \times 10^{-5} \mathrm{~kJ} \mathrm{~mol}^{-1} \mathrm{~nm}^{8}\right)$. This may reflect the small effect of dispersion interactions on the intermolecular interactions of water relative to electrostatic interactions, so these parameters may not hold truly physical values when there are other significant approximations in the model (i.e., induced polarization). Secondly, the model is sensitive to the damping coefficients and the selection of different values for these terms may yield different dispersion coefficients. Given the small magnitude of the $C_{8}$ coefficients for this model, pair-specific dispersion coefficients may be needed to describe dispersion interactions between water and other species. For liquid water, the use of a Buckingham-type repulsive term appears to be more significant than the inclusion of $C_{8}$ dispersion.

\section{CONCLUSIONS}

Using the ForceBalance code, a molecular mechanical model for liquid water was developed. This model differs from established water models because it replaces the LennardJones non-bonded potential with a more sophisticated potential that describes pairwise interatomic Pauli repulsion using a Buckingham-type exponential function and includes damped $C_{6}$ and $C_{8}$ dispersion interactions. The physical properties and radial distribution function 
are in excellent agreement with experimental data. The CustomNonBondForce feature of OpenMM was used to generate a GPU-accelerated implementation of this potential automatically. Benchmark simulations show this potential is only modestly more computationallyintensive than conventional Lennard-Jones-based potentials. The structural and physical properties of liquid water predicted by this model is comparable to models like TIP4P-FB, which employ the Lennard-Jones potential, although the more realistic description of the intermolecular potential of this model could open new opportunities to develop improved molecular mechanical force fields.

\section{ACKNOWLEDGMENTS}

The authors thank NSERC of Canada for funding through the Discovery Grants program (RGPIN-05795-2016), the School of Graduate Studies at Memorial University for a graduate fellowship, and Dr. Liqin Chen for a scholarship. Computational resources were provided by Compute Canada (RAPI: djk-615-ab). We gratefully acknowledge the support of NVIDIA Corporation with the donation of the Titan Xp GPU used for this research.

\section{REFERENCES}

${ }^{1}$ G. Lamoureux, A. D. MacKerell Jr., and B. Roux, J. Chem. Phys. 119, 5185 (2003).

${ }^{2}$ A. J. Lee and S. W. Rick, J. Chem. Phys. 134, 184507 (2011), https://doi.org/10.1063/1.3589419.

${ }^{3}$ M. L. Laury, L.-P. Wang, V. S. Pande, T. Head-Gordon, and J. W. Ponder, J. Phys. Chem. B 119, 9423 (2015), https://doi.org/10.1021/jp510896n.

${ }^{4}$ S. W. Rick, J. Comput. Chem. 37, $2060 \quad$ (2016), https://onlinelibrary.wiley.com/doi/pdf/10.1002/jcc.24426.

${ }^{5}$ V. P. Osinga, S. J. A. van Gisbergen, J. G. Snijders, and E. J. Baerends, J. Chem. Phys. 106, 5091 (1997).

${ }^{6}$ J. E. Jones, Proc. R. Soc. London, A 106, 463 (1924), http://rspa.royalsocietypublishing.org/content/106/738/463.full.pdf.

${ }^{7}$ R. M. Levy, L. Y. Zhang, E. Gallicchio, and A. K. Felts, J. Am. Chem. Soc. 125, 9523 (2003). 
${ }^{8}$ J. Dzubiella, J. M. J. Swanson, and J. A. McCammon, Phys. Rev. Lett. 96, 087802 (2006-03).

${ }^{9}$ C. Tan, Y.-H. Tan, and R. Luo, J. Phys. Chem. B 111, 12263 (2007).

${ }^{10}$ C. J. Fennell, C. Kehoe, and K. A. Dill, J. Am. Chem. Soc. 132, 234 (2010).

${ }^{11}$ R. A. Buckingham, Proc. R. Soc. Lond. A 168, 264 (1938).

${ }^{12}$ K. T. Tang and J. P. Toennies, J. Chem. Phys. 80, 3726 (1984), https://doi.org/10.1063/1.447150.

${ }^{13}$ C. L. Wennberg, T. Murtola, B. Hess, and E. Lindahl, J. Chem. Theory Comput. 9, 3527 (2013), http://dx.doi.org/10.1021/ct400140n.

${ }^{14}$ L.-P. Wang, T. J. Martinez, and V. S. Pande, J. Phys. Chem. Lett. 5, 1885 (2014), http://dx.doi.org/10.1021/jz500737m.

${ }^{15}$ T. Darden, D. York, and L. Pedersen, J. Chem. Phys. 98, 10089 (1993).

${ }^{16}$ M. R. Shirts, D. L. Mobley, J. D. Chodera, and V. S. Pande, J. Phys. Chem. B 111, 13052 (2007).

${ }^{17}$ P. Eastman, J. Swails, J. D. Chodera, R. T. McGibbon, Y. Zhao, K. A. Beauchamp, L.-P. Wang, A. C. Simmonett, M. P. Harrigan, C. D. Stern, R. P. Wiewiora, B. R. Brooks, and V. S. Pande, PLoS Comput. Biol. 13, 1 (2017).

18"OpenMM Transformer and B68 Input Files," https://github.com/mohebifar/ openmm-transformer, (accessed December 3, 2019).

${ }^{19}$ I.-C. Yeh and G. Hummer, J. Phys. Chem. B 108, 15873 (2004), http://dx.doi.org/10.1021/jp0477147.

${ }^{20}$ J. M. Sorenson, G. Hura, R. M. Glaeser, and T. Head-Gordon, J. Chem. Phys 113, 9149 (2000), https://doi.org/10.1063/1.1319615. 